\title{
Serum microRNA profiling and bioinformatics analysis of patients with type 2 diabetes mellitus in a Chinese population
}

\author{
ZE-MIN YANG ${ }^{1}$, LONG-HUI CHEN $^{2}$, MIN HONG $^{3}$, YING-YU CHEN $^{3}$, \\ XIAO-RONG YANG ${ }^{4}$, SI-MENG TANG ${ }^{1}$, QIAN-FA YUAN ${ }^{1}$ and WEI-WEN CHEN ${ }^{2}$
}

\begin{abstract}
${ }^{1}$ Department of Biochemistry and Molecular Biology, School of Basic Courses, Guangdong Pharmaceutical University, Guangzhou, Guangdong 510006; ${ }^{2} \mathrm{Pi}$-Wei Institute, Guangzhou University of Chinese Medicine, Guangzhou, Guangdong 510405; ${ }^{3}$ Department of Traditional Chinese Medicine; ${ }^{4}$ Clinical Laboratory, The First Affiliated Hospital of Guangdong Pharmaceutical University, Guangzhou, Guangdong 510080, P.R. China
\end{abstract}

Received December 31, 2015; Accepted December 19, 2016

DOI: $10.3892 / \mathrm{mmr} .2017 .6239$

\begin{abstract}
Type 2 diabetes mellitus (T2DM) is characterized by islet $\beta$-cell dysfunction and insulin resistance, which leads to an inability to maintain blood glucose homeostasis. Circulating microRNAs (miRNAs) have been suggested as novel biomarkers for T2DM prediction or disease progression. However, miRNAs and their roles in the pathogenesis of T2DM remain to be fully elucidated. In the present study, the serum miRNA expression profiles of T2DM patients in Chinese cohorts were examined. Total RNA was extracted from serum samples of 10 patients with T2DM and five healthy controls, and these was used in reverse-transcription-quantitative polymerase chain reaction analysis with the Exiqon PCR system of 384 serum/plasma miRNAs. A total of seven miRNAs were differentially expressed between the two groups (fold change $>3$ or $<0.33 ; \mathrm{P}<0.05)$. The serum expression levels of miR-455-5p, miR-454-3p, miR-144-3p and miR-96-5p were higher in patients with T2DM, compared with those of healthy subjects, however, the levels of miR-409-3p, miR-665 and $\mathrm{miR}-766-3 \mathrm{p}$ were lower. Hierarchical cluster analysis indicated that it was possible to separate patients with T2DM and control individuals into their own similar categories by these differential miRNAs. Target prediction showed that 97 T2DM candidate genes were potentially modulated by these seven miRNAs. Kyoto Encyclopedia of Genes and Genomes pathway analysis revealed that 24 pathways were enriched for these genes, and the majority of these pathways were enriched for the targets of induced and repressed miRNAs, among which insulin, adipocytokine and T2DM pathways, and several
\end{abstract}

Correspondence to: Professor Ze-Min Yang, Department of Biochemistry and Molecular Biology, School of Basic Courses, Guangdong Pharmaceutical University, 280 Waihuan Road East, Guangzhou, Guangdong 510006, P.R. China

E-mail: yzm3102001@gmail.com

Key words: serum microRNA, type 2 diabetes mellitus, quantitative polymerase chain reaction array, type 2 diabetes mellitus candidate genes, Kyoto Encyclopedia of Genes and Genomes pathway analysis cancer-associated pathways have been previously associated with T2DM. In conclusion, the present study demonstrated that serum miRNAs may be novel biomarkers for T2DM and provided novel insights into the pathogenesis of T2DM.

\section{Introduction}

Type 2 diabetes mellitus (T2DM), characterized by hyperglycemia, is one of the most prevalent metabolic disorders. The International Diabetes Federation estimates that $>400,000,000$ diabetic patients are expected by 2030, with over $50 \%$ of these being from Asia (1). Long-term hyperglycemia may lead to macrovascular diseases, including coronary artery disease, peripheral arterial disease and stroke, and microvascular complications, including diabetic nephropathy, neuropathy and retinopathy (2).

The pathogenesis of T2DM arises from the interplay of genetic, environmental and/or lifestyle factors, which lead to a decline in insulin sensitivity in the liver, adipose tissues and skeletal muscles, followed by chronic pancreatic $\beta$-cell dysfunction. Insulin resistance then increases insulin secretion (hyperinsulinemia) to maintain euglycemia. Subsequently, the progressive deterioration in insulin sensitivity and a reduction in pancreatic insulin secretion generate a state of relative insulin deficiency, resulting in chronic hyperglycemia and the onset of T2DM (3).

MicroRNAs (miRNAs) are endogenously expressed, evolutionarily conserved, small single-stranded, non-coding RNA molecules of 21-23 nucleotides, which function as regulators of gene expression by partially base-pairing to the 3 ' untranslated regions of their target mRNAs and destabilizing or inhibiting their translation (4). The latest estimates revealed that the human genome encodes $>1,600$ miRNA precursors, which can generate $>2,000$ mature miRNAs (www.mirbase.org), which control $\sim 50 \%$ of all mammalian protein-coding genes (5) and are involved in the biological processes of cell development, differentiation, metabolism, immunity, apoptosis and proliferation (4). The dysregulated expression of miRNAs in various tissues has been associated with a variety of diseases, including cancer (6,7), T2DM (8) and its complications (9). 
Serum or plasma miRNAs derived from various tissues/organs are released by several cellular release mechanisms. For example, mature miRNAs can bind to RNA-binding proteins or lipoproteins, or are loaded inside microvesicles or exosomes when they are to be released $(10,11)$. These circulating miRNAs may then be delivered to recipient cells, where they can regulate the translation of target genes, suggesting that serum or plasma miRNAs can serve as extracellular communicators (12). Furthermore, miRNAs levels in serum are stable, reproducible and consistent among individuals of the same ethnic background (10). The specific serum miRNA expression profile constitutes the fingerprint of a physiological or disease condition (10). Evidence from rat models shows that miRNA expression profiles in different tissues (pancreas, liver, adipose and skeletal muscle) share high similarity with those in blood samples (8). Therefore, circulating miRNAs are suggested as unique biomarkers, which are reflective and predictive of metabolic health and disorder (8). Furthermore, circulating miRNAs as novel biomarkers for DM and diabetic complications have been assessed in different studies $(11,13)$. For example, Zampetaki et al (14) revealed distinct profiles of serum miRNAs between patients with T2DM when compared with non-DM patients in a Bruneck cohort using miRNAs microarray technology. Similar findings were reported in Singapore by Karolina et al (15). Previous studies have also shown that certain specific serum miRNAs arre differentially expressed in patients with T2DM, compared with normal individuals, in China using reverse transcription-quantitative polymerase chain reaction (RT-qPCR) analysis (16-19). Furthermore, studies have shown that the majority of these candidate miRNAs are involved in regulating insulin secretion, insulin resistance, glucose homeostasis and/or lipid metabolism implicated in pathology of T2DM $(8,20-22)$.

Therefore, differentially expressed miRNAs in the blood may be suitable biomarkers for predicting T2DM or associated complications. However, miRNAs and their role in the etiology and pathogenesis of T2DM remain to be fully elucidated. Furthermore, inconsistent results have been obtained from different studies of T2DM-associated miRNAs, which may be due to ethnic variance of samples, different inclusion/exclusion criteria or different methods of miRNA analysis. A previous investigation revealed an ethnicity-specific miRNA profile of T2DM (23). Although RT-qPCR analysis is generally used to identify T2DM-associated miRNAs, certain studies have used high-throughput and microarray profiling, particulary those investigating Chinese cohorts. Increased knowledge of the circulating miRNA profiles of Chinese patients with T2DM can further contribute to current understanding of the development of T2DM with regards to different ethnic origins. Therefore, in the present study, an miRNA RT-qPCR array, combining the advantages of microarray and qPCR technology, was used to investigate differences in serum miRNA expression profiles between patients with T2DM and healthy subjects in Chinese cohorts. A total of seven potential miRNA biomarkers were identified in the patients with T2DM from the Chinese population. These miRNAs potentially regulated 97 T2DM candidate genes, which were enriched in several Kyoto Encyclopedia of Genes and Genomes (KEGG) pathways, including insulin, adipocytokine and T2DM pathways, elucidating the pathogenesis of T2DM.

\section{Materials and methods}

Ethics statement. The present study was approved by the Ethics Committee of The First Affiliated Hospital of Guangzhou University of Chinese Medicine (Guangzhou, China). All participants provided signed written informed consent prior to experiments.

Participants. A total of 10 patients with T2DM, comprising six women and four men aged 48-66 years old (58.2 \pm 7.7 years), were recruited from the First Affiliated Hospital of Guangzhou University of Chinese Medicine between October 2013 to December 2013. All patients were diagnosed by the criteria of the American Diabetes Association (24). Patients were excluded if they presented with severe diabetic complications, including stroke and/or other diseases in addition to T2DM, including infectious or inflammatory diseases, psychiatric conditions, serious somatic diseases or dyslipidemia. In addition, five healthy subjects, comprising three women and two mean aged 51-61 years old (56.4 \pm 3.7 years), were recruited as a control group through local advertisement. The healthy subjects were free of any endocrine diseases, including T2DM, and met the exclusion criteria for diabetes, which was then confirmed by Professor Ming Hong (The First Affiliated Hospital of Guangdong Pharmaceutical University) based on medical examination. These individuals were also excluded if they were overweight/obese, presented with a family history of diabetes or were on long-term medication.

Serum sample collection. Each participant, following a period of fasting between 7:00 a.m. and 9:00 a.m., had whole venous blood $(>3 \mathrm{ml})$ collected in a vacuum tube sans anti-coagulants. The samples were stored in a $4^{\circ} \mathrm{C}$ refrigerator for $1 \mathrm{~h}$ to allow complete blood coagulation. Subsequently, the yellow supernatant (serum) was centrifuged at 6,640 $\mathrm{x} g$ for $10 \mathrm{~min}$ at $4^{\circ} \mathrm{C}$ to remove residual cellular components. Every $250 \mu \mathrm{l}$ of serum was then packed in a frozen storage tube of RNase-free medium (Corning Incorporated, Corning, NY, USA) and stored at $-80^{\circ} \mathrm{C}$ prior to use. The whole procedure was completed within $2 \mathrm{~h}$ following blood sampling.

RNA isolation. Total RNA, including miRNA, was isolated from serum using TRIzol reagent (Invitrogen; Thermo Fisher Scientific, Inc., Waltham, MA, USA) according to the manufacturer's protocol. The concentration and purity of the RNA samples were determined using a NanoDrop ND-1000 spectrophotometer (Thermo Fisher Scientific, Inc.). RNA integrity was evaluated by denaturing agarose gel electrophoresis. RNA samples with a met $260 / 280$ value $>1.7$ and an RNA concentration $(20 \mu \mathrm{l})>60 \mu \mathrm{g} / \mu \mathrm{l}$ were used for the miRNA RT-qPCR array.

miRNA RT-qPCR array. For each sample, $\sim 20-25 \mathrm{ng}$ of total RNA containing miRNA was reverse transcribed into cDNA using the MicroRNA Reverse Transcription kit and the RT Primer Pools (Exiqon A/S, Vedbaek, Denmark) according to the manufacturer's protocol. The resulting cDNA served as a template for miRNA qPCR analysis in an ABI PRISM7900 system (Applied Biosystems; Thermo Fisher Scientific, Inc.) with the miRCURY LNA ${ }^{\mathrm{TM}}$ Universal RT microRNA PCR 
system, Ready-to-use Serum/Plasma Focus Human Panel I (Exiqon A/S; cat. no. 203886), which detected 372 human mature miRNAs in the serum samples from the 10 T2DM patients and five healthy subjects. Specifically, the resulting cDNA template was diluted 110 times in nuclease free water. The $10 \mu \mathrm{l}$ reaction volume contained $5 \mu \mathrm{l} \mathrm{SYBR}{ }^{\circledR}$-Green master mix, $1 \mu$ I PCR primer mix (Exiqon A/S) and $4 \mu 1$ diluted cDNA template. The amplification profile was denatured at $95^{\circ} \mathrm{C}$ for $10 \mathrm{~min}$, followed by 38 cycles of $95^{\circ} \mathrm{C}$ for $10 \mathrm{sec}$ and $60^{\circ} \mathrm{C}$ for $60 \mathrm{sec}$. Melting curve analyses were performed at the end of the PCR cycles. All procedures were performed according to the manufacturer's protocol.

Determination of differentially expressed miRNAs and cluster analysis. The raw quantification cycle $(\mathrm{Cq})$ values were obtained with the software supplied with the real-time qPCR instrument. The data was further analyzed with GenEx qPCR (Exiqon A/S) and SPSS 18.0 (SPSS, Inc., Chicago, IL, USA) analysis software. Briefly, the threshold value was set in the exponential amplification phase of the PCR. The Cq values were determined by the numbers of PCR cycles and threshold values. Undetectable data were assigned a default $\mathrm{Cq}$ value of 38. The $\mathrm{Cq}$ values were normalized by the delta $\mathrm{Cq}$ method with the housekeeping gene, SNORD38B, which had a stable $\mathrm{Cq}$ value in the serum of two groups. Differences in the delta Cq value between control and T2DM subjects were compared using Student's t-test (two-tailed). The relative expression levels (fold-change) of miRNAs between the two groups,

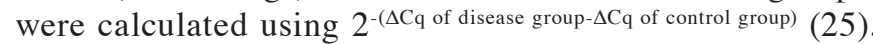
The miRNAs which matched $\mathrm{P}<0.05$ and fold change $>3.0$ or $<0.33$ were defined as differentially expressed miRNAs. Data are presented asrthe mean \pm standard deviation. Cluster analysis for differentially expressed miRNAs was performed using Multiple Experiment Viewer 4.9 software (TM4; http://www.jcvi.org/cms/research/software/) (26). The median center method was used to adjust genes/rows. Hierarchical clustering based on Pearson's correlation distance metric with average linkage was used to construct gene and sample trees.

miRNA target prediction and T2DM candidate gene search. To evaluate the functions of the differentially expressed miRNAs, miRNA target prediction was performed using the miRSystem database (version 20150312; http://mirsystem. cgm.ntu.edu.tw/) (27), which integrates the seven target gene prediction algorithms, Diana-microT (version 4.0), miRanda (August 2010 release), miRBridge (April 2010 release), PicTar (March 2007 release), PITA (August 2008 release), RNA22 (version 2.0) and Targetscan (version 6.0), and two experimentally validated databases, TarBase (version 7.0) and miRecords (November 2010 release). In the present study, only validated genes or miRNA-target interactions identified by at least three prediction programs were considered for further analysis. Target prediction was performed separately for upregulated and downregulated miRNAs.

To investigate the interactive association between target genes regulated by differentially expressed miRNAs and candidate genes for T2DM, the VENNY 1.0 tool (http://bioinfogp.cnb.csic.es/tools/venny_old/index.html) (28) was used to compare the lists of predicted targets of those miRNAs with a list of 563 candidate genes for T2DM using Venn diagrams. The list of 563 candidate genes was obtained from T2DM in T-the Text-mined Hypertension, Obesity and Diabetes candidate gene database (last updated on January 2th, 2014; http://bws.iis.sinica.edu.tw/THOD/) (29). This database provides lists of candidate genes for hypertension, obesity and diabetes, and is regularly updated by text-mining technologies, including a gene/disease identification system and a disease-gene relation extraction system, which is used to affirm the association of genes with the three diseases by domain experts. Furthermore, this database provides textual evidence of previous literature, disease-centric protein-protein interaction network, and integrated gene and single-nucleotide polymorphism information.

Functional annotation analysis of the predicted targets. The intersected gene list between predicted targets (upregulated and downregulated miRNAs) and candidate genes for T2DM were separately submitted to the Database for Annotation, Visualization, and Integrated Discovery (version 6.7) $(30,31)$, and the putative targets were annotated using KEGG pathway analysis (http://david.abcc.ncifcrf.gov/). The count threshold was set as two genes per annotation term. The threshold of EASE score, expressed as P-value in the present study and is a modified Fisher Exact P-value for gene-enrichment analysis, was set as 0.05 . $\mathrm{P}<0.05$ was considered to indicate increased enrichment in the annotation categories (https://david.ncifcrf. gov/helps/functional_annotation.html, \#summary).

\section{Results}

Differential miRNA expression in serum between patients with T2DM and healthy subjects. An miRNA qPCR array containing 372 human serum mature miRNAs was used to compare the serum miRNA expression profiles between 10 patients with T2DM and five healthy subjects. A total of 24 miRNAs showed significant differences $(\mathrm{P}<0.05)$ in expression levels between the two groups. Of these, seven miRNAs matched the fold change $>3.0$ or $<0.33$ (shown as red and green in Fig. 1). The fold changes of the seven miRNAs are presented in Fig. 2. The present study found that four miRNAs (hsa-miR-455-5p, hsa-miR-454-3p, hsa-miR-144-3p and hsa-miR-96-5p) and three miRNAs (hsa-miR-409-3p, hsa-miR-665 and hsa-miR-766-3p) were upregulated and downregulated, respectively. Furthermore, hierarchical cluster analysis showed that it was possible to separate patients with T2DM and control subjects into similar categories via the seven miRNAs, as all patients were clustered together and separated from the control subjects (Fig. 3).

T2DM candidate genes potentially regulated by the differentially expressed miRNAs. The predicted target genes of the seven differentially expressed miRNAs were identified using the miRSystem database. The results indicated 2,005 (list 3) and 565 (list 1) putative target genes for the upregulated and downregulated miRNAs, respectively (Fig. 4). The VENNY tool (29) was then used to analyze the overlaps among the predicted target genes (list 1 and list 3), and T2DM candidate genes (list 2). The Venn diagrams showed that, of the 563 T2DM candidate genes, a total of 97 T2DM candidate genes 
were regulated by the differentially expressed miRNAs identified in the present study, with 29 and 82 genes being predicted by the downregulated and upregulated miRNAs, respectively, and 14 by predicted by both (Fig. 4).

Potential functions of the differentially expressed miRNAs. KEGG pathway analysis was introduced to annotate the T2DM candidate genes predicted by the differentially expressed miRNAs. The results showed that the T2DM candidate genes predicted by upregulated and downregulated miRNAs were enriched in 19 and 13 KEGG pathways, respectively (Fig. 5). Furthermore, eight KEGG pathways were shared by both, which comprised insulin, adipocytokine, mammalian target of rapamycin (mTOR), long-term depression, hypertrophic cardiomyopathy (HCM), cancer (melanoma and glioma) and focal adhesion signaling pathways. Of the 24 enriched pathways, three KEGG pathways were directly associated with the pathomechanism of T2DM. These were T2DM, insulin and adipocytokine signaling pathways, which included 19 T2DM candidate genes, which were regulated by the differentially expressed miRNAs. The interactions between these T2DM targets and the seven differentially expressed miRNAs are shown in Fig. 6. Of the 19 targets, seven targets were regulated by downregulated miRNAs, 12 targets were regulated by upregulated miRNAs, and three targets were regulated by both, which were RPS6KB1, SHC1 and peroxisome proliferator-activated receptor $\gamma$, coactivator $1 \alpha$ (PPARGC1A). Considering the fold-changes of the miRNAs for these three targets, RPS6KB1 and PPARGC1A were potentially repressed and SHC1 was overexpressed by corresponding miRNAs (Figs. 2 and 7).

In the insulin signaling pathway (Fig. 7A), upregulated miRNAs inhibited insulin receptor substrate (IRS) 1 , further reduced antilipolysis via phosphodiesterase 3B, (PDE3B; cGMP-inhibited) and protein synthesis via ribosomal protein S6 kinase, $70 \mathrm{kDa}$, polypeptide 1 (RPS6KB1), increased lipogenesis via protein kinase, AMP-activated, $\alpha 1$ and 2 catalytic subunits (PRKAA1 and 2), and affected glycolysis/gluconeogenesis via forkhead box O1 (FOXO1) and PPARGC1A. The upregulated miRNAs decreased cell proliferation, differentiation and protein synthesis via $\mathrm{Src}$ homology 2 domain containing, transforming protein 1 (SHC1) and mitogen-activated protein kinase kinase 1 (MAP2K1). By contrast, the downregulated miRNAs predominantly affected glycolysis/gluconeogenesis via IRS2, v-akt murine thymoma viral oncogene homolog 1 (AKT1), PPARGC1A and glucose-6-phosphatase, catalytic subunit (G6PC). The downregulated miRNAs also increased protein synthesis via SHC1 and RPS6KB1. Combining these two aspects, patients with T2DM exhibited insulin resistance, characteristic of a metabolic disorder of glucose and lipid homeostasis.

In the adipocytokine and T2DM signaling pathways (Fig. 7B), upregulated miRNAs affected the insulin signaling pathway via IRS1, tumor necrosis factor $\alpha(\mathrm{TNF} \alpha)$, tumor necrosis factor receptor superfamily, member 1B (TNFRSF1B) and suppressor of cytokine signaling 3 (SOCS3), reduced mitochondrion $\beta$-oxidation via PPARGC1A and peroxisome proliferator-activated receptor $\alpha$ (PPARA), and decreased glucose uptake via PRKAA1/2 and solute carrier family 2 , member 1 (SLC2A1). In addition, upregulated miRNAs inhibited insulin secretion via calcium voltage-gated channel

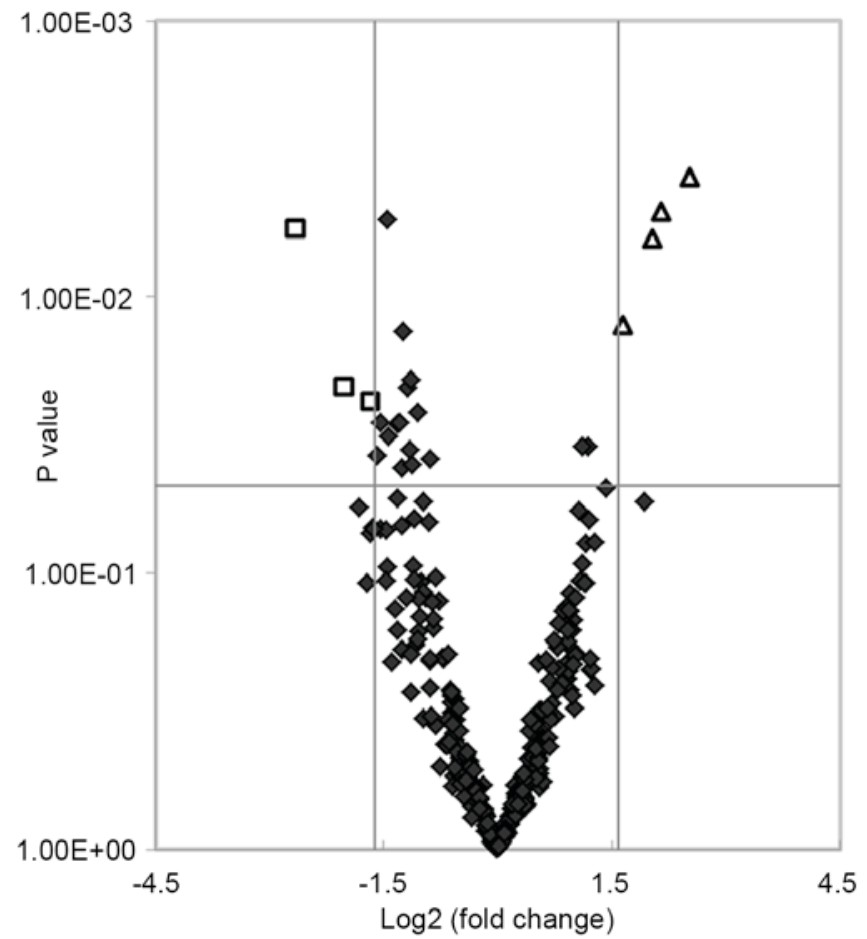

Figure 1. Scatterplot of differentially expressed miRNAs in serum between patients with type 2 diabetes mellitus and control subjects. $\log _{2}$ fold changes and corresponding P-values of all miRNAs in the array were obtained to construct the scatterplot. Negative values of $\log _{2}$ fold changes indicated downregulated miRNAs and positive values indicated upregulated miRNAs. Squares represent differentially expressed miRNAs with a fold change $>3.0$ or $<0.33$ and $\mathrm{P}<0.05$; triangles represent indicate upregulated miRNAs; diamonds represent all other miRNAs in the array. miRNA, microRNA.

subunit $\alpha 1 \mathrm{E}$ (CACNA1E), a voltage dependent $\mathrm{R}$ type calcium channel. The downregulated miRNAs increased mitochondrion $\beta$-oxidation via PPARGC1A and leptin (LEP), and increase gluconeogenesis via G6PC.

\section{Discussion}

In the present study, a qPCR array, which included 372 human mature miRNAs, was used to examine differences in serum miRNA expression profiles between patients with T2DM and healthy subjects. A total of seven differentially expressed miRNAs were identified, which improved stratification of the two groups. Target gene prediction indicated that 97 T2DM candidate genes were regulated by these miRNAs. KEGG functional annotation showed that these T2DM candidate genes were significantly enriched in the insulin, adipocytokine and T2DM signaling pathways, as well as several other pathways.

As the pathogenesis of T2DM remains to be fully elucidated, biomarkers used for the early detection and identification of at risk individuals have the potential to improve patient quality of life by providing improved management. The levels of serum miRNAs derived from various tissues/organs are stable, reproducible and consistent among individuals of the same ethnic origin. Additionally, the expression profiles of serum miRNAs better reflect underlying pathological/physiologic processes $(10,32)$ and have been used extensively in various types of cancer $(6,7)$ and metabolic syndromes (15), 


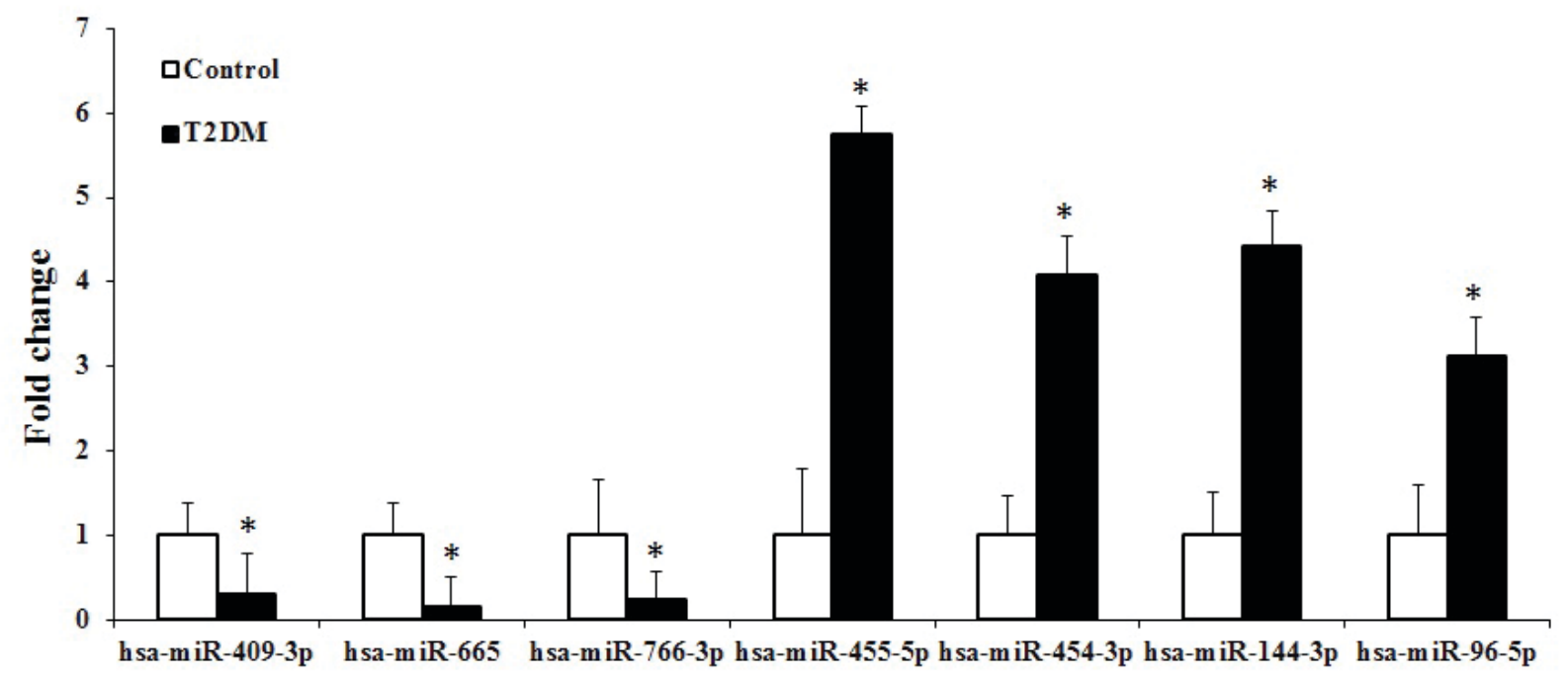

Figure 2. Differential expression of miRNAs in serum between patients with T2DM and control subjects. The expression levels of serum hsa-miR-455-5p, hsa-miR-454-3p, hsa-miR-144-3p and hsa-miR-96-5p were upregulated in the patients with T2DM, compared with those in the control subjects, whereas hsa-miR-409-3p, hsa-miR-665 and hsa-miR-766-3p were downregulated. "P<0.05 vs. control. miRNA, microRNA; T2DM, type 2 diabetes mellitus.

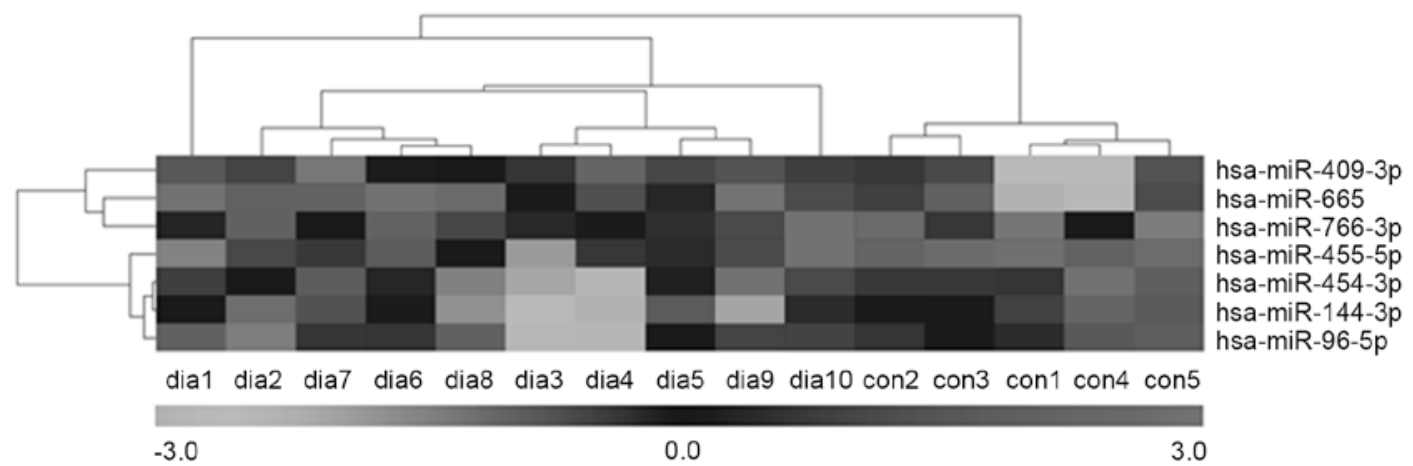

Figure 3. Cluster dendrogram of the differential miRs in serum between patients with type 2 diabetes mellitus and control subjects. Each column represents an individual sample and each row represents one miRNA. Black indicates lower expression and white indicates higher expression. miR, microRNA; dia, diabetes; con, control.

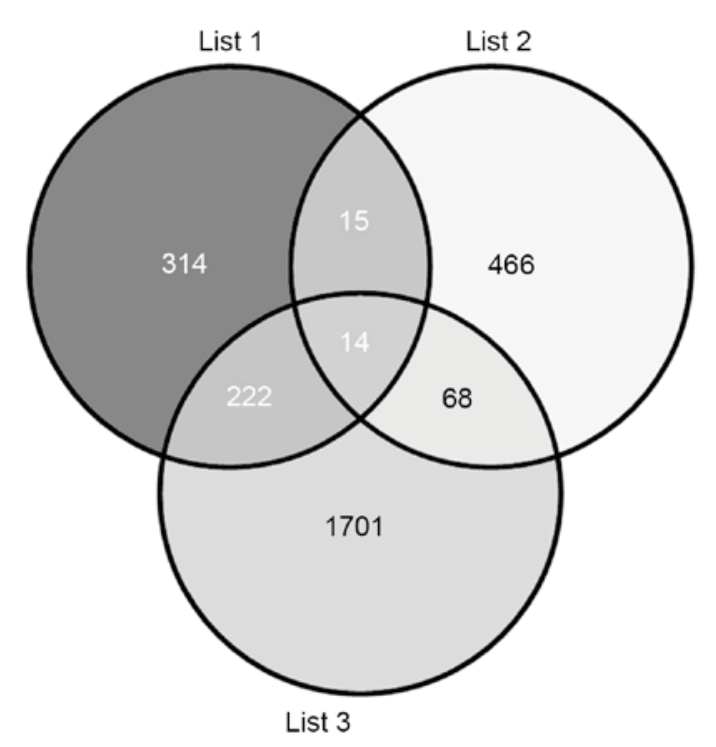

Figure 4. Venn diagrams between the arget genes of the seven differentially expressed miRNAs and T2DM candidate genes. Lists 1 and 3 represent targets of downregulated and upregulated miRNAs, respectively, and list 2 indicates T2DM candidate genes. miRNA, microRNA; T2DM, type 2 diabetes mellitus. including T2DM $(8,11)$. Furthermore, accumulating evidence has identified several serum miRNAs, which regulate insulin signaling, glucose and lipid metabolism, as implicated in T2DM pathology $(8,20-22)$. Thus, serum miRNAs may serve as novel biomarkers for T2DM and also assist in explaining its pathogenesis.

Several studies have assessed the differences in serum or plasma miRNA expression between patients with T2DM and healthy non-diabetic subjects. Using miRNA microarray profiling confirmed by qPCR, Zampetaki et al (14) first identified low plasma levels of miR-15a, miR-29b, miR-126 and miR-223, and high levels of miR-28-3p in patients with T2DM, compared with non-diabetic individuals in Bruneck, Italy. Karolina et al (15) found upregulation in the levels of miR-27a, miR-150, miR-192, miR-320a and miR-375 in the blood and exosomes of patients with T2DM, compared with healthy controls in Singapore. Using qPCR analysis of specific miRNAs, Kong et al (18) also found that seven candidate miRNAs (miR-9, miR-29a, miR-30d, miR-34a, miR-124a, miR-146a and miR-375) were significantly upregulated in serum from patients newly diagnosed with T2DM, compared with T2DM-susceptible individuals and normal glucose 

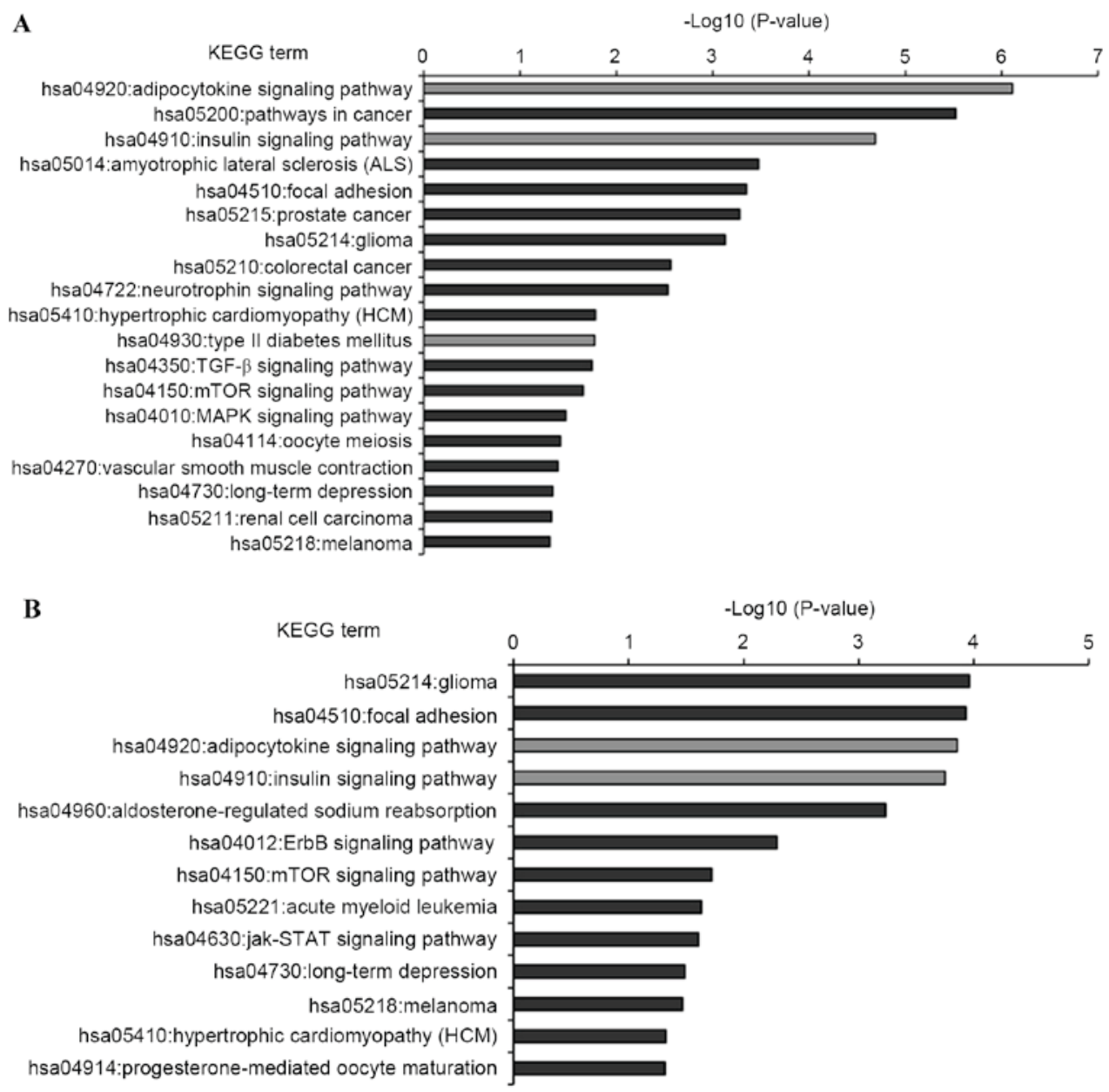

Figure 5. KEGG pathway functional annotations of type 2 diabetes mellitus candidate genes regulated by the differentially expressed miRNAs. (A) KEGG pathway of upregulated miRNAs; (B) KEGG pathway of downregulated miRNAs. Enrichment scores (P-values) of each pathway provided by the Database for Annotation, Visualization, and Integrated Discovery annotation tool are showed as -log10 (P-values). miR, microRNA; KEGG, Kyoto Encyclopedia of Genes and Genomes.

tolerance in a Chinese cohort. These pioneering studies demonstrated the potential of miRNAs as biomarkers for T2DM, although with mixed results. In the present study, seven differently expressed miRNAs were identified. The upregulation of miR-144-3p has been supported in several other reports. Wang et al (23) found that a higher expression of miR-144 in plasma was significantly associated with T2DM in Sweden. Similar results were reported by Yang et al (33), and Zhu and Leung (34) showed that the upregulation of circulating miR-144 may be a potential biomarker for T2DM in a meta-analysis of controlled profiling studies. Karolina et al (8) found that miR-144 was significantly increased in blood samples from a T2DM rat model. In addition, the upregulation of miR-144 was shared among patients with T1DM,T2DM and gestational diabetes mellitus in peripheral blood mononuclear cells, and expression was higher in muscles of patients with T2DM, compared with healthy individuals (35). Compared with the findings of the present study, low expression levels of miR-96 were reported by Yang et al (19) in the serum of patients with T2DM, compared with normal glucose tolerance controls. To the best of our knowledge, none of the other differentially expressed miRNAs identified in the present study have been reported in previous studies associated with T2DM. Thus, the present study may have identified novel dysregulated miRNAs in patients with T2DM, compared with control individuals, at least in the Chinese population examined.

The present study also predicted the T2DM candidate genes, which were potentially regulated by the seven differential miRNAs. Of the 563 T2DM candidate genes, 97 genes were identified (Fig. 4), which may be important in explaining the role of these miRNAs in the pathogenesis of T2DM. KEGG functional annotation of these targets showed that several pathways were potentially modulated by these upregulated and/or downregulated miRNAs (Fig. 5). The majority of these pathways have been previously associated with T2DM, including insulin and adipocytokine signaling pathways, T2DM, pathways in cancer, focal adhesion, and hypertrophic cardiomyopathy (as described below). These findings may provide novel insights into the complex molecular mechanisms involved in T2DM.

Relative insulin deficiency and insulin resistance are important characteristics in the development of T2DM pathogenesis. In the present study, three signaling pathways (insulin, adipocytokine and T2DM) showed marked enrichment with the 19 T2DM candidate genes modulated by the downregulated and upregulated miRNAs (Figs. 6 and 7), which have been implicated in insulin secretion and function in T2DM. For insulin secretion, the upregulation of miR-96-5p represses 


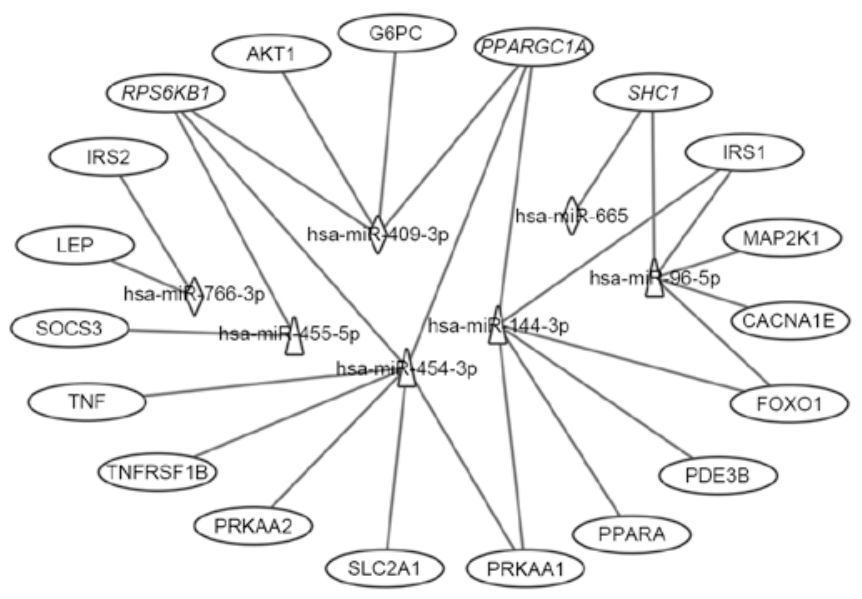

Figure 6. Interaction networks of the differential miRNAs and their T2DM target genes associated with type II diabetes mellitus, insulin and adipocytokine signaling pathways. Triangles represent upregulated miRNAs; diamonds represent downregulated miRNAs and ovals represent target genes for T2DM. Italics (RPS6KB1, PPARGC1A and SHC1) indicate genes targeted by downregulated and upregulated miRNAs. miR, microRNA T2DM, type 2 diabetes mellitus; CACNA1E, calcium voltage-gated channel subunit $\alpha 1 \mathrm{E}$; TNFRSF1B, tumor necrosis factor $\alpha$ receptor superfamily, member 1B; IRS, insulin receptor substrate; SOCS3, suppressor of cytokine signaling 3; PPARGC1A, peroxisome proliferator-activated receptor $\gamma$, coactivator 1 $\alpha$; PPARA, peroxisome proliferator-activated receptor $\alpha$; PRKAA1, protein kinase, AMP-activated, $\alpha 1$; AKT1, v-akt murine thymoma viral oncogene homolog 1; SLC2A1, solute carrier family 2, member 1; G6PC, glucose-6-phosphatase, catalytic subunit; LEP, leptin; SHC1, Src homology 2 domain containing, transforming protein 1; MAP2K1, mitogen-activated protein kinase kinase 1; RPS6KB1, ribosomal protein S6 kinase, 70 kDa, polypeptide 1; PDE3B. phosphodiesterase 3B; FOXO1, forkhead box O1.

CACNA1E and then results in impaired insulin secretion. Similar reports have shown that miR-96 negatively regulates insulin exocytosis by granuphilin/SLP4 (20). Dysregulated insulin and adipocytokine signaling pathways can affect glucose, lipid, and protein metabolism, which result in insulin resistance. Specifically, these identified miRNAs may dysregulate the glycolysis/gluconeogenesis process via the targeting of IRS1, IRS2, FOXO1, PPARGC1A, AKT1 and G6PC, and repress glucose uptake via PRKAA1/2 and SLC2A1. They may also dysregulate the process of lipogenesis via the targeting of IRS1, IRS2, PDE3B and PRKAA1/2, and inhibit mitochondrial $\beta$-oxidation via PPARA and PPARGC1A. In addition, these miRNAs may dysregulate protein synthesis processes via the targeting of IRS1, IRS2, SHC1, MAP2K1 and RPS6KB1. In addition, TNF $\alpha$, TNFRSF1B and LEP indirectly affect insulin signaling pathways and lipolysis processes. Karolina et al (8) experimentally demonstrated that IRS1 is the target of miR-144, and that increased circulating levels of miR-144 are correlated with downregulation of its predicted target, IRS1, at the mRNA and protein levels. Similar results were reported by Yang et al (33). Furthermore, Jeong et al (36) and Wang et al (37) revealed that IRS1 is also the target of miR-96. FOXO1 was experimentally demonstrated in several investigations $(38,39)$ to serve as the target of miR-96. None of the other interactions of the target-miRNAs identified in the present study have been reported previously. Of note, previous reports have shown that several miRNAs identified in the present study were involved in carbohydrate and lipid metabolism. Hu et al (40) and Ramírez et al (41) revealed that
miR-144 regulates cholesterol metabolism and plasma levels of high-density lipoprotein, and promotes pro-inflammatory cytokine production. Fu et al (42) found that miR-144 regulates carbohydrate and lipid metabolism by inhibiting isocitrate dehydrogenase 2, which acts as key enzyme of the tricarboxylic acid cycle. Similar reports have also demonstrated functions of miR-96, which controls selective high-density lipoprotein cholesterol and cholesteryl ester uptake, and regulates endogenous lipid synthesis $(22,43,44)$. In addition, Milagro et al $(45)$ found that the expression of miR-766 is correlated with weight loss. Therefore, these miRNAs may be able to regulate lipid metabolism through the insulin, adipocytokine and T2DM pathways. Additionally, the dysregulation of carbohydrate and lipid metabolism modulated by the identified miRNAs may be an important pathogenic mechanism of T2DM.

Evidence of an association between DM and cancer has been sugested, although without a definitive conclusion. Previous reports have shown that DM and insulin resistance are risk factors for gastric, hepatocellular and prostate cancer (46). In addition, breast cancer, colon cancer (47), melanoma (48), renal cell carcinoma (49) and pancreatic cancer (50) have been implicated in the progression of T2DM. In the present study, and in agreement with the previous studies, several signaling pathways were found to be involved. Previous studies have also shown that these miRNAs are associated with increased risk of cancer. For example, miR-144-3p exerts antitumor effects in glioblastoma (51), and is a diagnostic marker for breast cancer (52), follicular thyroid cancer (53), laryngeal carcinoma (54) and papillary thyroid carcinoma (55). Similar results also revealed an association between miRNA-96-5p and several types of cancer, including breast cancer (56), colorectal carcinoma (57), epithelial ovarian cancer (58), pancreatic carcinoma (59) and prostate cancer (60). miR-454-3p can enhance cellular radiosensitivity in renal carcinoma cells by inhibiting the expression of BTG anti-proliferation factor 1 (61). miR-455-5p can promote melanoma growth and metastasis through inhibition of the tumor suppressor gene, cytoplasmic polyadenylation element binding protein 1 (62). In additiob, miR-455-5p was identified as a molecular signature associated with anaplastic large cell lymphoma (63), basal cell carcinoma (64), endometrial serous adenocarcinomas (65) and laryngeal cancer (66). miR-409-3p suppresses the invasion and metastasis of colorectal (67) and bladder cancer (68), but promotes the tumorigenesis of human prostate cancer (69) and gastric cancer (70). Furthermore, plasma miR-409-3p serves as a promising biomarker for the early detection of breast cancer (71) and colorectal cancer (72). The downregulation of miR-665 may be closely associated with the invasive metastatic and chemoresistance of gastric signet ring cell carcinoma (73). However, no report has shown an association between miR-766-3p and cancer. Taken together, the findings of the present study corroborated with previous studies, which linked T2DM and cancer. It is possible that a number of the patients with T2DM in the present study were at risk of cancer.

In the present study, the predicted target genes were also significantly enriched in the focal adhesion and hypertrophic cardiomyopathy pathways. Wang et al (74) found that the focal adhesion pathway is significantly dysregulated in the progression of T2DM by assessing 

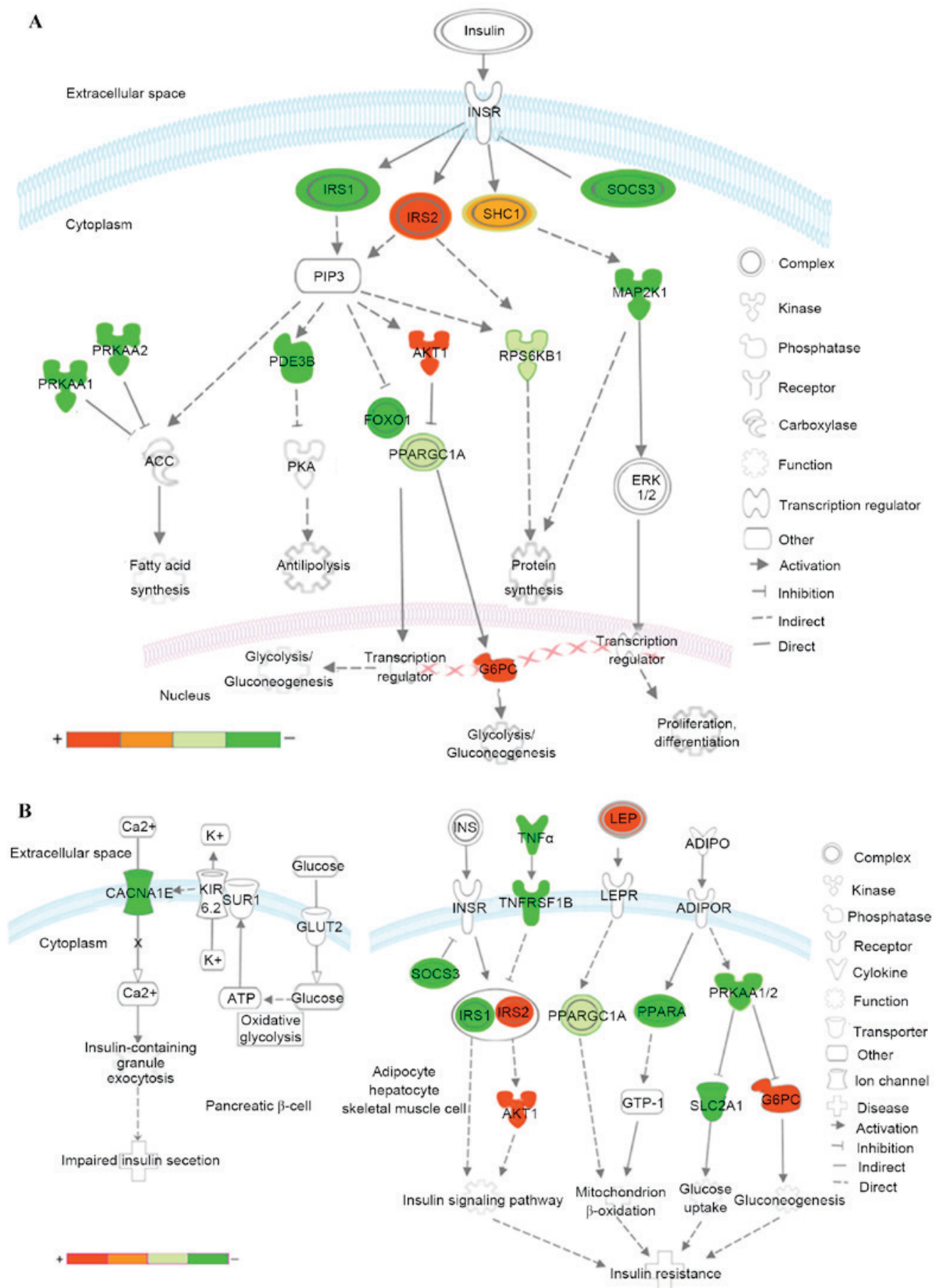

Figure 7. Kyoto Encyclopedia of Genes and Genomes pathways associated with T2DM and T2DM candidate genes regulated by the differentially expressed miRNAs. (A) Insulin signaling pathway (B) Pathways of adipocytokine signaling and type II diabetes mellitus. Red represents overexpressed genes targeted by downregulated miRNAs, and green represents repressed genes targeted by upregulated miRNAs. Orange and pea green represent genes targeted by both downregulated and upregulated miRNAs, although the former was primarily overexpressed and the latter was repressed. miR, microRNA; T2DM, type 2 diabetes mellitus; CACNA1E, calcium voltage-gated channel subunit $\alpha 1 \mathrm{E}$; KIR, inward rectifying potassium channel; SUR1, sulfonylurea receptor 1; GLUT2, glucose transporter 2; TNF $\alpha$, tumor necrosis factor $\alpha$; TNFRSF1B, receptor superfamily, member 1B; IRS, insulin receptor substrate; INSR, INS receptor; SOCS3, suppressor of cytokine signaling 3; PPARGC1A, peroxisome proliferator-activated receptor $\gamma$, coactivator 1 $\alpha$; PPARA, peroxisome proliferator-activated receptor $\alpha$; PRKAA1/2, protein kinase, AMP-activated, $\alpha 1$ and 2; AKT1, v-akt murine thymoma viral oncogene homolog 1; GTP-1, glutamate pyruvate transaminase-1; SLC2A1, solute carrier family 2, member 1; G6PC, glucose-6-phosphatase, catalytic subunit; LEP, leptin; LEPR, leptin receptor; ADIPO, adiponectinp; ADIPOR, adiponectin receptor; SHC1, Src homology 2 domain containing, transforming protein 1; MAP2K1, mitogen-activated protein kinase kinase 1; RPS6KB1, ribosomal protein S6 kinase, 70kDa, polypeptide 1; ACC, acetyl-CoA carboxylase; PDE3B, phosphodiesterase 3B; FOXO1, forkhead box O1; ERK, extracellular signal-regulated kinase.

differentially expressed genes between human pancreatic islets with T2DM and normal islets. Similar results were reported in female visceral and subcutaneous adipose, and in male visceral adipose and skeletal muscle of patients with
T2DM (75). In terms of HCM pathway, asymmetric left ventricular hypertrophy and impairment in diastolic function were important characteristics of HCM. Dinh et al (76) and Shigematsu et al (77) found that insulin resistance and 
glycemic abnormalities were associated with the deterioration of left ventricular diastolic function. Okayama et al (78) also revealed that the presence of obstructive coronary stenosis and the magnitude of left ventricular hypertrophy were associated with the presence of diabetes, triglyceride levels and estimated glomerular filtration rate. In addition, the results of previous studies have shown that the mTOR signaling pathway, also enriched in the present study, is implicated in left ventricular remodeling, myocardial infarction and hypertrophic cardiomyopathy $(79,80)$. Therefore, the findings of the present study suggested that the abnormal pathway of focal adhesion may be a pathological feature of T2DM, and that aberrant expression of miRNAs may also induce diabetic cardiomyopathy by targets implicated in the HCM pathway.

In conclusion, the present study identified seven differentially expressed miRNAs by using an miRNA qPCR array. These miRNAs clearly discriminated patients with T2DM from healthy subjects and offer potential as suitable biomarkers for T2DM by assessing for abnormal expression. In addition, target gene prediction revealed that a total of 97 T2DM candidate genes may be regulated by these differential miRNAs. The results of the present study were concordant with those of previous reports, to a certain extent, in that several biological pathways previously implicated in T2DM were potentially modulated by the seven miRNAs, including insulin and adipocytokine signaling pathways, T2DM and several cancer-associated pathways. Taken together, the results of the present study may provide novel insight into the possibility that circulating miRNAs can be used as potential biomarkers for T2DM, which assists in improving current understanding of the pathomechanism and biological pathways underlying T2DM.

\section{Acknowledgements}

The authors would like to thank Dr Zhang-Zhi Zhu and Dr Sai-Mei Li of the First Affiliated Hospital of Guangzhou University of Chinese Medicine for support in participant recruitment, and Li-Ping Zhang of the Art Department of Guangdong Light Industry School, China, for assisting with figures. The authors would also like to thank John Lees (Monell Chemical Senses Center, Philadelphia, PA, USA), for language editing. This study was supported by the National Natural Science Foundation of China (grant no. 81102703 to Professor Ze-Min Yang), the Science and Technology Planning Project of Guangdong Province of China (grant no. 2013A032500005 to Professor Ze-Min Yang), the Administration of Traditional Chinese Medicine of Guangdong Province of China (grant no. 20123001 to Professor Wei-Wen Chen), the Special Funds from Central Finance of China in Support of the Development of Local Colleges and Universities in 2013 (grant no. 338 to Professor Wei-Wen Chen), the Natural Science Foundation for Fostering of Guangdong Pharmaceutical University of China (grant no. GYFYLH201303 to Professor Ze-Min Yang), and the South China Chinese Medicine Collaborative Innovation Center (grant no. A1-AFD01514A05 to Professor Wei-Wen Chen). Dr Long-Hui Chen received support from the China Scholarship Council as a joint PhD student at the University of Pennsylvania, USA.

\section{References}

1. Whiting DR, Guariguata L, Weil C and Shaw J: IDF diabetes atlas: Global estimates of the prevalence of diabetes for 2011 and 2030. Diabetes Res Clin Pract 94: 311-321, 2011.

2. Fowler MJ: Microvascular and macrovascular complications of diabetes. Clinical Diabetes 26: 77-82, 2008.

3. Ferrannini E, Gastaldelli A and Iozzo P: Pathophysiology of prediabetes. Med Clin North Am 95: 327-339, vii-viii, 2011.

4. Bartel DP: MicroRNAs: Genomics, biogenesis, mechanism, and function. Cell 116: 281-297, 2004.

5. Krol J, Loedige I and Filipowicz W: The widespread regulation of microRNA biogenesis, function and decay. Nat Rev Genet 11: 597-610, 2010.

6. Rossing M, Borup R, Henao R, Winther O, Vikesaa J, Niazi O, Godballe C, Krogdahl A, Glud M, Hjort-Sørensen C, et al: Down-regulation of microRNAs controlling tumourigenic factors in follicular thyroid carcinoma. J Mol Endocrinol 48: 11-23, 2012.

7. Sand M, Skrygan M, Sand D, Georgas D, Hahn SA, Gambichler T, Altmeyer P and Bechara FG: Expression of microRNAs in basal cell carcinoma. Br J Dermatol 167: 847-855, 2012.

8. Karolina DS, Armugam A, Tavintharan S, Wong MT, Lim SC, Sum CF and Jeyaseelan K: MicroRNA 144 impairs insulin signaling by inhibiting the expression of insulin receptor substrate 1 in type 2 diabetes mellitus. PLoS One 6: e22839, 2011.

9. Kantharidis P, Wang B, Carew RM and Lan HY: Diabetes complications: The microRNA perspective. Diabetes 60: 1832-1837, 2011.

10. Chen X, Ba Y, Ma L, Cai X, Yin Y, Wang K, Guo J, Zhang Y, Chen J, Guo X, et al: Characterization of microRNAs in serum: A novel class of biomarkers for diagnosis of cancer and other diseases. Cell Res 18: 997-1006, 2008.

11. Guay C and Regazzi R: Circulating microRNAs as novel biomarkers for diabetes mellitus. Nat Rev Endocrinol 9: 513-521, 2013.

12. Creemers EE, Tijsen AJ and Pinto YM: Circulating microRNAs: Novel biomarkers and extracellular communicators in cardiovascular disease? Circ Res 110: 483-495, 2012.

13. Chien HY, Lee TP, Chen CY, Chiu YH, Lin YC, Lee LS and Li WC: Circulating microRNA as a diagnostic marker in populations with type 2 diabetes mellitus and diabetic complications. J Chin Med Assoc 78: 204-211, 2015.

14. Zampetaki A, Kiechl S, Drozdov I, Willeit P, Mayr U, Prokopi M, Mayr A, Weger S, Oberhollenzer F, Bonora E, et al: Plasma microRNA profiling reveals loss of endothelial miR-126 and other microRNAs in type 2 diabetes. Circ Res 107: 810-887, 2010.

15. Karolina DS, Tavintharan S, Armugam A, Sepramaniam S, Pek SL, Wong MT, Lim SC, Sum CF and Jeyaseelan K: Circulating miRNA profiles in patients with metabolic syndrome. J Clin Endocrinol Metab 97: E2271-E2276, 2012.

16. Zhang T, Lv C, Li L, Chen S, Liu S, Wang C and Su B: Plasma miR-126 is a potential biomarker for early prediction of type 2 diabetes mellitus in susceptible individuals. Biomed Res Int 2013: 761617, 2013.

17. Rong Y, Bao W, Shan Z, Liu J, Yu X, Xia S, Gao H, Wang X, Yao P, Hu FB and Liu L: Increased microRNA-146a levels in plasma of patients with newly diagnosed type 2 diabetes mellitus. PLoS One 8: e73272, 2013.

18. Kong L, Zhu J, Han W, Jiang X, Xu M, Zhao Y, Dong Q, Pang Z, Guan Q, Gao L, et al: Significance of serum microRNAs in pre-diabetes and newly diagnosed type 2 diabetes: A clinical study. Acta Diabetol 48: 61-69, 2011.

19. Yang Z, Chen H, Si H, Li X, Ding X, Sheng Q, Chen P and Zhang H: Serum miR-23a, a potential biomarker for diagnosis of pre-diabetes and type 2 diabetes. Acta Diabetol 51: 823-831, 2014.

20. Chen H, Lan HY, Roukos DH and Cho WC: Application of microRNAs in diabetes mellitus. J Endocrinol 222: R1-R10, 2014.

21. Dehwah MA, Xu A and Huang Q: MicroRNAs and type 2diabetes/obesity. J Genet Genomics 39: 11-18, 2012.

22. Jeon TI, Esquejo RM, Roqueta-Rivera M, Phelan PE, Moon YA, Govindarajan SS, Esau CC and Osborne TF: An SREBP-responsive microRNA operon contributes to a regulatory loop for intracellular lipid homeostasis. Cell Metab 18: 51-61, 2013

23. Wang X, Sundquist J, Zöller B, Memon AA, Palmér K, Sundquist $\mathrm{K}$ and Bennet L: Determination of 14 Circulating microRNAs in Swedes and Iraqis with and without Diabetes Mellitus Type 2. PLoS One 9: e86792, 2014. 
24. American Diabetes Association: Economic costs of diabetes in the U.S. in 2012. Diabetes Care 36: 1033-1046, 2013.

25. Livak KJ and Schmittgen TD: Analysis of relative gene expression data using real-time quantitative PCR and the 2(-Delta Delta C (T)) Method. Methods 25: 402-408, 2001.

26. Saeed AI, Sharov V, White J, Li J, Liang W, Bhagabati N, Braisted J, Klapa M, Currier T, Thiagarajan M, et al: TM4: A free, open-source system for microarray data management and analysis. Biotechniques 34: 374-378, 2003.

27. Lu TP, Lee CY, Tsai MH, Chiu YC, Hsiao CK, Lai LC and Chuang EY: miRSystem: An integrated system for characterizing enriched functions and pathways of microRNA targets. PLoS One 7: e42390, 2012.

28. Oliveros JC: VENNY. An interactive tool for comparing lists with Venn Diagrams. 2007. http://bioinfogp.cnb.csic. es/tools/venny/index.html. Accessed November 20, 2013.

29. Dai HJ, Wu JC, Tsai RT, Pan WH and Hsu WL: T-HOD: A literature-based candidate gene database for hypertension, obesity and diabetes. Database (Oxford) 2013: bas061, 2013.

30. Huang DW, Sherman BT and Lempicki RA: Bioinformatics enrichment tools: Paths toward the comprehensive functional analysis of large gene lists. Nucleic Acids Res 37: 1-13, 2009.

31. Huang DW, Sherman BT and Lempicki RA: Systematic and integrative analysis of large gene lists using DAVID bioinformatics resources. Nat Protoc 4: 44-57, 2009.

32. Gilad S, Meiri E, Yogev Y, Benjamin S, Lebanony D, Yerushalmi N, Benjamin H, Kushnir M, Cholakh $\mathrm{H}$ Melamed N, et al: Serum microRNAs are promising novel biomarkers. PLoS One 3: e3148, 2008.

33. Yang S, Zhao J, Chen Y and Lei M: Biomarkers associated with ischemic stroke in diabetes mellitus patients. Cardiovasc Toxicol 16: 213-222, 2016.

34. Zhu $\mathrm{H}$ and Leung SW: Identification of microRNA biomarkers in type 2 diabetes: A meta-analysis of controlled profiling studies. Diabetologia 58: 900-911, 2015.

35. Collares CV, Evangelista AF, Xavier DJ, Rassi DM, Arns T, Foss-Freitas MC, Foss MC, Puthier D, Sakamoto-Hojo ET, Passos GA and Donadi EA: Identifying common and specific microRNAs expressed in peripheral blood mononuclear cell of type 1 , type 2 , and gestational diabetes mellitus patients. BMC Res Notes 6: 491, 2013.

36. Jeong HJ, Park SY, Yang WM and Lee W: The induction of miR-96 by mitochondrial dysfunction causes impaired glycogen synthesis through translational repression of IRS-1 in SK-Hep1 cells. Biochem Biophys Res Commun 434: 503-508, 2013.

37. Wang Y, Luo H, Li Y, Chen T, Wu S and Yang L: hsa-miR-96 up-regulates MAP4K1 and IRS1 and may function as a promising diagnostic marker in human bladder urothelial carcinomas. Mol Med Rep 5: 260-265, 2012.

38. Yu JJ, Wu YX, Zhao FJ and Xia SJ: miR-96 promotes cell proliferation and clonogenicity by down-regulating of FOXO1 in prostate cancer cells. Med Oncol 31: 910, 2014

39. Fendler A, Jung M, Stephan C, Erbersdobler A, Jung K and Yousef GM: The antiapoptotic function of miR-96 in prostate cancer by inhibition of FOXO1. PLoS One 8: e80807, 2013.

40. Hu YW, Hu YR, Zhao JY, Li SF, Ma X, Wu SG, Lu JB, Qiu YR, Sha YH, Wang YC, et al: An agomir of miR-144-3p accelerates plaque formation through impairing reverse cholesterol transport and promoting pro-inflammatory cytokine production. PLoS One 9: e94997, 2014.

41. Ramírez CM, Rotllan N, Vlassov AV,Dávalos A,Li M, Goedeke L, Aranda JF, Cirera-Salinas D, Araldi E, Salerno A, et al: Control of cholesterol metabolism and plasma high-density lipoprotein levels by microRNA-144. Circ Res 112: 1592-1601,2013.

42. Fu X, Huang X, Li P, Chen W and Xia M: 7-Ketocholesterol inhibits isocitrate dehydrogenase 2 expression and impairs endothelial function via microRNA-144. Free Radic Biol Med 71: $1-15,2014$

43. Wang L, Jia XJ, Jiang HJ, Du Y, Yang F, Si SY and Hong B: MicroRNAs 185, 96, and 223 repress selective high-density lipoprotein cholesterol uptake through posttranscriptional inhibition. Mol Cell Biol 33: 1956-1964, 2013.

44. Meyer JM, Graf GA and van der Westhuyzen DR: New developments in selective cholesteryl ester uptake. Curr Opin Lipidol 24 386-392, 2013.

45. Milagro FI, Miranda J, Portillo MP, Fernandez-Quintela A, Campión J and Martínez JA: High-throughput sequencing of microRNAs in peripheral blood mononuclear cells: Identification of potential weight loss biomarkers. PLoS One 8: e54319, 2013.
46. Best LG, García-Esquinas E, Yeh JL, Yeh F, Zhang Y, Lee ET, Howard BV, Farley JH, Welty TK, Rhoades DA, et al: Association of diabetes and cancer mortality in American Indians: The strong heart study. Cancer Causes Control 26: 1551-1560, 2015.

47. Onitilo AA, Stankowski RV, Berg RL, Engel JM, Glurich I, Williams GM and Doi SA: Type 2 diabetes mellitus, glycemic control, and cancer risk. Eur J Cancer Prev 23: 134-140, 2014

48. Qi L, Qi X, Xiong H, Liu Q, Li J, Zhang Y, Ma X, Wu N, Liu Q and Feng L: Type 2 diabetes mellitus and risk of malignant melanoma: A systematic review and meta-analysis of cohort studies. Iran J Public Health 43: 857-866, 2014.

49. Vavallo A, Simone S, Lucarelli G, Rutigliano M, Galleggiante V, Grandaliano G, Gesualdo L, Campagna M, Cariello M, Ranieri E, et al: Pre-existing type 2 diabetes mellitus is an independent risk factor for mortality and progression in patients with renal cell carcinoma. Medicine (Baltimore) 93: e183, 2014

50. Brodovicz KG, Kou TD, Alexander CM, O'Neill EA, Engel SS, Girman CJ and Goldstein BJ: Impact of diabetes duration and chronic pancreatitis on the association between type 2 diabetes and pancreatic cancer risk. Diabetes Obes Metab 14: 1123-1128, 2012.

51. Lan F, Yu H, Hu M, Xia T and Yue X: miR-144-3p exerts anti-tumor effects in glioblastoma by targeting c-Met. J Neurochem 135: 274-286, 2015.

52. Chang CW, Wu HC, Terry MB and Santella RM: microRNA expression in prospectively collected blood as a potential biomarker of breast cancer risk in the BCFR. Anticancer Res 35: 3969-3977, 2015

53. Stokowy T, Eszlinger M, Świerniak M, Fujarewicz K, Jarząb B, Paschke R and Krohn K: Analysis options for high-throughput sequencing in miRNA expression profiling. BMC Res Notes 7: 144,2014

54. Lu ZM, Lin YF, Jiang L, Chen LS, Luo XN, Song XH, Chen SH and Zhang SY: Micro-ribonucleic acid expression profiling and bioinformatic target gene analyses in laryngeal carcinoma. Onco Targets Ther 7: 525-533, 2014.

55. Swierniak M, Wojcicka A, Czetwertynska M, Stachlewska E, Maciag M, Wiechno W, Gornicka B, Bogdanska M, Koperski L, de la Chapelle A and Jazdzewski K: In-depth characterization of the microRNA transcriptome in normal thyroid and papillary thyroid carcinoma. J Clin Endocrinol Metab 98: E1401-E1409, 2013.

56. Matamala N, Vargas MT, González-Cámpora R, Miñambres R, Arias JI, Menéndez P, Andrés-León E, Gómez-López G, Yanowsky K, Calvete-Candenas J, et al: Tumor MicroRNA expression profiling identifies circulating MicroRNAs for early breast cancer detection. Clin Chem 61: 1098-1096, 2015.

57. Kara M, Yumrutas O, Ozcan O, Celik OI, Bozgeyik E, Bozgeyik I and Tasdemir S: Differential expressions of cancer-associated genes and their regulatory miRNAs in colorectal carcinoma. Gene 567: 81-86, 2015.

58. Wang L, Zhu MJ, Ren AM, Wu HF, Han WM, Tan RY and Tu RQ: A ten-microRNA signature identified from a genome-wide microRNA expression profiling in human epithelial ovarian cancer. PLoS One 9: e96472, 2014.

59. Li C, Du X, Tai S, Zhong X, Wang Z, Hu Z, Zhang L, Kang P, Ji D, Jiang X, et al: GPC1 regulated by miR-96-5p, rather than miR-182-5p, in inhibition of pancreatic carcinoma cell proliferation. Int J Mol Sci 15: 6314-6327, 2014.

60. Larne O, Martens-Uzunova E, Hagman Z, Edsjö A, Lippolis G, den Berg MS, Bjartell A, Jenster G and Ceder Y: miQ-a novel microRNA based diagnostic and prognostic tool for prostate cancer. Int J Cancer 132: 2867-2875, 2013

61. Wu X, Ding N, Hu W, He J, Xu S, Pei H, Hua J, Zhou G and Wang J: Down-regulation of BTG1 by miR-454-3p enhances cellular radiosensitivity in renal carcinoma cells. Radiat Oncol 9: 179, 2014

62. Shoshan E, Mobley AK, Braeuer RR, Kamiya T, Huang L, Vasquez ME, Salameh A, Lee HJ, Kim SJ, Ivan C, et al: Reduced adenosine-to-inosine miR-455-5p editing promotes melanoma growth and metastasis. Nat Cell Biol 17: 311-321, 2015.

63. Liu C, Iqbal J, Teruya-Feldstein J, Shen Y, Dabrowska MJ, Dybkaer K, Lim MS, Piva R, Barreca A, Pellegrino E, et al: MicroRNA expression profiling identifies molecular signatures associated with anaplastic large cell lymphoma. Blood 122: 2083-2092, 2013

64. Sand M, Skrygan M, Sand D, Georgas D, Hahn SA, Gambichler T, Altmeyer P and Bechara FG: Expression of microRNAs in basal cell carcinoma. Br J Dermatol 167: 847-855, 2012. 
65. Hiroki E, Akahira J, Suzuki F, Nagase S, Ito K, Suzuki T, Sasano $\mathrm{H}$ and Yaegashi $\mathrm{N}$ : Changes in microRNA expression levels correlate with clinicopathological features and prognoses in endometrial serous adenocarcinomas. Cancer Sci 101: 241-249, 2010.

66. Saito K, Inagaki K, Kamimoto T, Ito Y, Sugita T, Nakajo S, Hirasawa A, Iwamaru A, Ishikura $\mathrm{T}$, Hanaoka $\mathrm{H}$, et al: MicroRNA-196a is a putative diagnostic biomarker and therapeutic target for laryngeal cancer. PLoS One 8: e71480, 2013

67. Bai R, Weng $\mathrm{C}$, Dong $\mathrm{H}$, Li S, Chen $\mathrm{G}$ and $\mathrm{Xu} \mathrm{Z}$ : MicroRNA-409-3p suppresses colorectal cancer invasion and metastasis partly by targeting GAB1 expression. Int $\mathrm{J}$ Cancer 137: 2310-2322, 2015

68. Xu X, Chen H, Lin Y, Hu Z, Mao Y, Wu J, Xu X, Zhu Y, Li S, Zheng $X$ and Xie L: MicroRNA-409-3p inhibits migration and invasion of bladder cancer cells via targeting c-Met. Mol Cells 36: 62-88, 2013.

69. Josson S, Gururajan M, Hu P, Shao C, Chu GY, Zhau HE, Liu C, Lao K, Lu CL, Lu YT, et al: miR-409-3p/-5p promotes tumorigenesis, epithelial-to-mesenchymal transition, and bone metastasis of human prostate cancer. Clin Cancer Res 20: 4636-4646, 2014.

70. Li C, Nie H, Wang M, Su L, Li J, Yu B, Wei M, Ju J, Yu Y, Yan M, et al: MicroRNA-409-3p regulates cell proliferation and apoptosis by targeting PHF10 in gastric cancer. Cancer Lett 320: 189-197, 2012

71. Cuk K, Zucknick M, Heil J, Madhavan D, Schott S, Turchinovich A, Arlt D, Rath M, Sohn C, Benner A, et al: Circulating microRNAs in plasma as early detection markers for breast cancer. Int J Cancer 132: 1602-1612, 2013.

72. Wang S, Xiang J, Li Z, Lu S, Hu J, Gao X, Yu L, Wang L, Wang J, Wu Y, et al: A plasma microRNA panel for early detection of colorectal cancer. Int J Cancer 136: 152-161, 2015.
73. Chen J, Sun D, Chu H, Gong Z, Zhang C, Gong B, Li Y, Li N and Jiang L: Screening of differential microRNA expression in gastric signet ring cell carcinoma and gastric adenocarcinoma and target gene prediction. Oncol Rep 33: 2963-2971, 2015.

74. Wang Q, Zhao Z, Shang J and Xia W: Targets and candidate agents for type 2 diabetes treatment with computational bioinformatics approach. J Diabetes Res 2014: 763936, 2014.

75. Jain P, Vig S, Datta M, Jindel D, Mathur AK, Mathur SK and Sharma A: Systems biology approach reveals genome to phenome correlation in type 2 diabetes. PLoS One 8: e53522, 2013.

76. Dinh W, Lankisch M, Nick1 W, Scheyer D, Scheffold T, Kramer F, Krahn T, Klein RM, Barroso MC and Füth R: Insulin resistance and glycemic abnormalities are associated with deterioration of left ventricular diastolic function: A cross-sectional study. Cardiovasc Diabetol 9: 63, 2010.

77. Shigematsu Y, Hamada M, Nagai T, Nishimura K, Inoue K, Suzuki J, Ogimoto A and Higaki J: Risk for atrial fibrillation in patients with hypertrophic cardiomyopathy: Association with insulin resistance. J Cardiol 58: 18-25, 2011.

78. Okayama S, Soeda T, Kawakami R, Takami Y, Somekawa S, Ueda T, Sugawara Y, Matsumoto T, Sung JH, Nishida T, et al: Evaluation of coronary artery disease and cardiac morphology and function in patients with hypertrophic cardiomyopathy, using cardiac computed tomography. Heart Vessels 30: 28-35, 2015.

79. Diniz GP, Carneiro-Ramos MS and Barreto-Chaves ML: Angiotensin type 1 receptor mediates thyroid hormone-induced cardiomyocyte hypertrophy through the Akt/GSK-3beta/mTOR signaling pathway. Basic Res Cardiol 104: 653-667, 2009.

80. Marin TM, Keith K, Davies B, Conner DA, Guha P, Kalaitzidis D, Wu X, Lauriol J, Wang B, Bauer M, et al: Rapamycin reverses hypertrophic cardiomyopathy in a mouse model of LEOPARD syndrome-associated PTPN11 mutation. J Clin Invest 121: 1026-1043, 2011. 